\title{
Genome-wide profiling of IncRNA expression patterns in patients with acute promyelocytic leukemia with differentiation therapy
}

\author{
JIAN YU ${ }^{1 *}$, XIAO-LING GUO ${ }^{2 *}$, YUAN-YUAN BAI $^{1}$, JUN-JUN YANG $^{1}$, \\ XIAO-QUN ZHENG ${ }^{1}$, JI-CHEN RUAN ${ }^{3}$ and ZHAN-GUO CHEN ${ }^{1}$ \\ ${ }^{1}$ Department of Clinical Laboratory, ${ }^{2}$ Research Center, ${ }^{3}$ Department of Pediatric Hematology, \\ The Second Affiliated Hospital and Yuying Children's Hospital of Wenzhou Medical University, \\ Wenzhou, Zhejiang 325000, P.R. China
}

Received January 11, 2018; Accepted June 18, 2018

DOI: 10.3892/or.2018.6521

\begin{abstract}
Long non-coding RNAs (lncRNAs) are crucial factors in acute promyelocytic leukemia (APL) cell differentiation. However, their expression patterns and regulatory functions during all-trans-retinoic acid (ATRA)-induced APL differentiation remain to be fully elucidated. The profile of dysregulated IncRNAs between three bone marrow (BM) samples from patients with APL post-induction and three BM samples from untreated matched controls was examined with the Human Transcriptome Array 2.0. The dysregulated lncRNA expression of an additional 27 APL BM samples was validated by reverse transcription-quantitative
\end{abstract}

Correspondence to: Dr Zhan-Guo Chen, Department of Clinical Laboratory, The Second Affiliated Hospital and Yuying Children's Hospital of Wenzhou Medical University, 109 Xueyuan Xi Road, Wenzhou, Zhejiang 325000, P.R. China

E-mail: steve0577@126.com

Dr Ji-Chen Ruan, Department of Pediatric Hematology, The Second Affiliated Hospital and Yuying Children's Hospital of Wenzhou Medical University, 109 Xueyuan Xi Road, Wenzhou, Zhejiang 325000, P.R. China

E-mail: ruanjichen@163.com

*Contributed equally

Abbreviations: APL, acute promyelocytic leukemia; lncRNAs, long non-coding RNAs; ATRA, all-trans-retinoic acid; mRNA, messenger RNA; BM, bone marrow; RT-qPCR, reverse transcription-quantitative polymerase chain reaction; ATO, arsenic trioxide; AML, acute myeloid leukemia; MBMCs, mononuclear bone marrow cells; TFs, transcription factors; FC, fold change; KEGG, Kyoto Encyclopedia of Genes and Genomes; GO, Gene Ontology; EEA1, early endosome antigen 1; ZNF564, zinc finger protein 564; ZNF44, zinc finger protein 44; E2F1, E2F transcription factor 1; EBF1, early B cell factor 1; E2F6, E2F transcription factor 6

Key words: acute promyelocytic leukemia, long non-coding RNAs, differentiation therapy, expression pattern polymerase chain reaction (RT-qPCR) analysis. The lncRNA functions were predicted through co-expressed messenger RNA (mRNA) annotations. Co-expressed IncRNA-mRNA networks were constructed to analyze the functional pathways. In total, 825 lncRNAs and 1,218 mRNAs were dysregulated in the treated APL BM group, compared with the untreated APL BM group. The expression of 10 selected lncRNAs was verified by RT-qPCR analysis. During APL differentiation, NONHSAT076891 was the most upregulated lncRNA, whereas TCONS_00022632-XLOC_010933 was the most downregulated. Functional analysis revealed that several lncRNAs may exert activities in biological pathways associated with ATRA-induced APL differentiation through cis and/or trans regulation of mRNAs. The findings of the present study assist in explaining the contributions of IncRNAs in APL myeloid differentiation and improve current knowledge on the potential mechanisms regarding dysregulated IncRNA expression in ATRA-induced APL differentiation.

\section{Introduction}

Acute promyelocytic leukemia (APL) is distinguished by a specific $\mathrm{t}(15 ; 17)$ chromosomal translocation, contributing to the expression of the oncoprotein PML-RAR $\alpha$, which counteracts myeloid differentiation and facilitates APL-initiating cell self-renewal $(1,2)$. The dominant-negative effect of the PML-RAR $\alpha$ oncoprotein, which antagonizes the myeloid differentiation process, can be reversed by pharmacological doses of all-trans-retinoic acid (ATRA) (3). The combined therapy of ATRA and arsenic trioxide (ATO), which eliminates APL by activating PML-RAR $\alpha$ degradation, demonstrates efficacy as an APL therapy and markedly improves the prognosis of patients with APL (4). However, ATO and ATRA induce irreversible resistance, and certain patients with APL ultimately succumb to treatment-resistant diseases $(5,6)$. Therefore, the unifying mechanisms required for myeloid differentiation and response to therapy in APL require further investigation.

Long non-coding RNAs (IncRNAs), which are in excess of 200 nucleotides, are involved in diverse biological 
processes including epigenetic regulation, chromosome imprinting, transcription, splicing, translation, cell-cycle control, and differentiation (7). They have been functionally coupled to cancer and cellular differentiation. The investigation of lncRNA expression and function may contribute to the current understanding of leukemogenesis, and the identification of novel therapeutic targets and seminal posttranscriptional factors associated with resistance to chemotherapy. Consequently, the global aberrant expression of lncRNAs that occurs during myeloid differentiation can assist in enhancing the efficacy of current APL therapies and identifying novel therapeutic targets for chemotherapies. IncRNAs have been considered as prognostic and diagnostic molecular biomarkers for acute myeloid leukemia (AML) $(8,9)$. Previous investigations have demonstrated that lncRNAs are crucial in myeloid differentiation and APL therapy (10-12). HOTAIRM1, a myeloid-specific lncRNA, has been defined as a key factor in ATRA-induced APL differentiation $(11,13)$. Furthermore, HOTAIRM1 can promote the degradation of PML-RAR $\alpha$ via a pathway associated with autophagy during myeloid cell differentiation or ATRA-induced APL differentiation (10). This suggests that aberrant lncRNA expression may be underlying targets for APL therapy and indicators for response to APL therapy. Several lncRNAs have been identified in APL-associated myeloid differentiation (10-13); however, additional key IncRNAs and their functions in regulating myeloid maturation require characterization in the APL-associated myeloid differentiation transcriptome.

NB4 cells represent a suitable cell model to investigate changes in IncRNA expression between APL cells and their terminally differentiated counterparts. System analysis of the transcriptome has been beneficial for the identification of various pathways or cascades at the transcriptome level associated with ATRA/ATO-induced cell differentiation (14). These are common in the absence of the complicated and dynamic intracorporeal synergy between ATRA and ATO in APL therapy, which may affect cell survival and response to therapy. Although it has been suggested that lncRNAs are involved in ATRA/ATO-induced extracorporeal APL differentiation, further understanding of the IncRNA landscape in ATRA/ATO-induced intracorporeal APL differentiation is required.

The aim of the present study was to examine lncRNA profiles and regulatory functions in ATRA-based targeted therapy for APL differentiation. The lncRNA and messenger RNA (mRNA) profiles were compared in three post-induction bone marrow (BM) samples from patients with APL and pre-induction (untreated) matched controls via whole transcriptome microarray. Subsequently, 10 dysregulated IncRNAs were selected and verified by reverse transcription-quantitative polymerase chain reaction (RT-qPCR) analysis in another 27 APL BM samples. In addition, the functions of dysregulated lncRNAs were predicted via their co-expressed mRNAs. The findings identified the lncRNA landscape for myeloid differentiation in APL and revealed potential mechanisms occurring due to dysregulated lncRNA expression in ATRA-induced APL differentiation; this may provide underlying targets for APL therapy and lncRNA biomarkers for APL responses to therapy.
Table I. Primary characteristics of patients with APL.

\begin{tabular}{lc}
\hline Characteristic & $\mathrm{n}(\%)$ \\
\hline Patients & 30 \\
Sex & \\
Male & $18(60.0)$ \\
Female & $12(40.0)$ \\
Age (years) & \\
Median (range) & $40(19-67)$ \\
WHO classification & \\
APL with t(15;17)(q22;q12); PML-RARa & $30(100.0)$ \\
Transcripts of PML-RARa & $30(100.0)$ \\
PML-RARa bcr1 & $18(60.0)$ \\
PML-RARa bcr2 & $1(3.3)$ \\
PML-RARa bcr3 & $11(36.7)$ \\
Received ATRA-based therapy & $30(100.0)$ \\
Paired BM post-induction obtained & $30(100.0)$
\end{tabular}

APL, acute promyelocytic leukemia; BM, bone marrow; WHO, World Health Organization; lncRNA, long non-coding RNA.

\section{Materials and methods}

Patient profiles. A total of 30 patients with APL who received ATRA-based targeted therapy at the Second Affiliated Hospital and Yuying Children's Hospital of Wenzhou Medical University (Wenzhou, China) between January 2014 and December 2016 were recruited for the present study. None of the patients received chemotherapy prior to targeted therapy. Written informed consent was collected from all the patients in conformity with the Declaration of Helsinki, and the present study obtained permission from the Ethics Committee of Wenzhou Medical University. The BM samples from the patients with APL at diagnosis and corresponding BM samples from patients with APL post-induction were collected in BD Vacutainer Heparin tubes (BD Biosciences, San Diego, CA, USA). Patient diagnoses were determined according to the 2016 World Health Organization criteria (15). The patients with APL were treated according to the International APL guidelines (15). Among the 60 samples, three paired samples (comprising three post-induction samples and three pre-induction samples) were used for IncRNA microarray analysis and the other samples were used for RT-qPCR analysis. The primary characteristics of the patients with APL are listed in Table I.

Sample collection and RNA extraction. The mononuclear $\mathrm{BM}$ cells (MBMCs) were isolated from $\sim 2 \mathrm{ml}$ heparinized BM samples using density gradient medium centrifugation (800 x g, for $20 \mathrm{~min}$ at room temperature). Subsequently, $1 \times 10^{7}$ cells were resuspended in TRIzol ${ }^{\circledR}$ reagent (Invitrogen; Thermo Fisher Scientific, Inc., Waltham, MA, USA) and temporarily stored at $-80^{\circ} \mathrm{C}$ until further analysis. Total RNA was extracted from the MBMCs according to the manufacturer's protocol and dissolved in $100 \mu 1$ nuclease-free water. The RNA yield was measured using a NanoDrop ND-2000 
Table II. Reverse transcription-quantitative polymerase chain reaction primers for lncRNAs.

\begin{tabular}{|c|c|c|}
\hline lncRNA primary ID & Primer sequence $\left(5^{\prime}-3^{\prime}\right)$ & Product (bp) \\
\hline NONHSAT121385 & $\begin{array}{l}\text { Forward: TACATGTTCCTGGTGAAAT } \\
\text { Reverse: GGCATCAAGTATGTCTCT }\end{array}$ & 108 \\
\hline ENST00000469418 & $\begin{array}{l}\text { Forward: CAATTTACGGCTGGACGTTT } \\
\text { Reverse: GAAAGGAATGCTGGGAAACA }\end{array}$ & 128 \\
\hline ENST00000424415 & $\begin{array}{l}\text { Forward: ATATTGAGATAGGAGGATGG } \\
\text { Reverse: GGCTTCTTCTAGGATAAGT }\end{array}$ & 107 \\
\hline NR_003186 & $\begin{array}{l}\text { Forward: TACATGTTCCTGGTGAAAT } \\
\text { Reverse: ATCTTTGGGCATCAAGTA }\end{array}$ & 115 \\
\hline ENST00000536425 & $\begin{array}{l}\text { Forward: TTGAATAATCCTAAATTATACATAC } \\
\text { Reverse: TCATAGTGACTAAATTGAATAAGTACCAAA }\end{array}$ & 78 \\
\hline NONHSAT061249 & $\begin{array}{l}\text { Forward: AGGATCGCTTGAGATGCAGT } \\
\text { Reverse: GCTACCGCTCTCAAGTTTGG }\end{array}$ & 110 \\
\hline TCONS_00017553-XLOC_008249 & $\begin{array}{l}\text { Forward: GTGTCTGTGTGTACAGAA } \\
\text { Reverse: ACATTCCATACACACAAAC }\end{array}$ & 187 \\
\hline TCONS_12_00030950-XLOC_12_015963 & $\begin{array}{l}\text { Forward: GTTGGAAGATGAAGGAAC } \\
\text { Reverse: ATCACTGTGTAAAGGACTA }\end{array}$ & 114 \\
\hline NONHSAT076891 & $\begin{array}{l}\text { Forward: GGATCTCCCCTGTGTTCTCA } \\
\text { Reverse: GACCAGGTAGTGGGGGAAGT }\end{array}$ & 146 \\
\hline TCONS_00022632-XLOC_010933 & $\begin{array}{l}\text { Forward: TCTTCCACGTAACAACCA } \\
\text { Reverse: CTGACAGTGTCTTCCATA }\end{array}$ & 124 \\
\hline
\end{tabular}

lncRNA, long non-coding RNA.

spectrophotometer from Thermo Fisher Scientific, Inc. and the RNA integrity was assessed using an Agilent 2100 bioanalyzer system (Agilent Technologies, Inc., Santa Clara, CA, USA). When the 28S:18S ratio was ascertained and the RNA integrity number (RIN) of each sample was assigned; RNA samples with a $28 \mathrm{~S}: 18 \mathrm{~S}$ ratio $\geq 0.7$ and $\mathrm{RIN} \geq 7.0$ were further analyzed.

lncRNA and mRNA microarray expression profiling. RNA from each sample ( $200 \mathrm{ng}$ ) was applied for lncRNA and mRNA microarray analyses using Cluster 3.0 (http://bonsai. hgc.jp/ mdehoon/software/cluster/software.htm). Gene expression was analyzed using an Affymetrix GeneChip ${ }^{\circledR}$ Human Transcriptome Array 2.0 (Affymetrix, Inc., Santa Clara, CA, USA). The microarray contained 67,539 probes for 22,829 human IncRNAs and 44,710 human mRNAs, which were derived from eight authoritative databases, including RefSeq, Ensembl, UCSC, MGC, nocode, IncRNAdb, Broad Institute, TUCP catalogue and Human Body Map lincRNAs. Additionally, the microarray contained probes for small non-coding RNAs, but not microRNAs, and the majority of these were small nuclear RNAs and small nucleolar RNAs. The array experiments and computational analysis were performed according to the manufacturer's protocol (Affymetrix, Inc.). The raw data were extracted and standardized using the GeneChip Command Console software 4.0 and Expression Console software 1.3.1 from Affymetrix, Inc. Additional data processing was performed with GeneSpring software 12.5
(Agilent Technologies, Inc.). Dysregulated IncRNAs or mRNAs defined by an absolute value of fold change $(F C) \geq 2.0$ and $\mathrm{P} \leq 0.05$ (Student's t-test) were selected for further analysis. The dysregulated mRNAs were categorized into different Gene Ontology (GO) and Kyoto Encyclopedia of Genes and Genomes (KEGG) annotation groups. The lncRNA chip experiments were performed in the laboratory at Shanghai OE Biotech Co., Ltd. (Shanghai, China).

$R T$-qPCR verification of 10 dysregulated lncRNAs. The lncRNA and $m R N A$ expression were analyzed using FastStart Universal SYBR-Green Master Mix (Rox) from Roche Diagnostics (Indianapolis, IN, USA) and an ABI ViiA ${ }^{\mathrm{TM}} 7$ Real-Time PCR system from Applied Biosystems (Thermo Fisher Scientific, Inc.). Briefly, the total RNA was transcribed into cDNA using a Transcriptor First Strand cDNA Synthesis kit (Roche Diagnostics) as per the manufacturer's protocol. PCR amplification was performed in a $25 \mu \mathrm{l}$ reaction containing $1 \mu \mathrm{l}$ of cDNA template ( $10 \mathrm{ng}), 12.5 \mu \mathrm{l}$ of FastStart Universal SYBR-Green Master Mix (Rox), $10.5 \mu$ of nuclease-free water, and $0.5 \mu \mathrm{l}$ of each pair of primers (Shanghai GeneCore BioTechnologies Co., Ltd., Shanghai, China). The RT-qPCR primers for the lncRNAs are listed in Table II. The reaction conditions were as follows: $95^{\circ} \mathrm{C}$ for $10 \mathrm{~min}$, followed by 40 cycles of $95^{\circ} \mathrm{C}$ for $15 \mathrm{sec}$ and $60^{\circ} \mathrm{C}$ for $45 \mathrm{sec}$. All experiments were repeated three times in parallel. The expression of lncRNAs was further standardized to the GAPDH gene and calculated using the $2^{-\Delta \Delta \mathrm{Cq}}$ method (16). 
Microarray results analysis and functional prediction of selected dysregulated lncRNAs. The identification of the overall functional distributions for the dysregulated lncRNAs identified in the experiment were performed as follows. Briefly, the co-expressed mRNAs for each dysregulated lncRNA were first calculated, and functional enrichment analysis for the set of co-expressed mRNAs was then performed. The enriched functional terms served as the predicted functional term for a given IncRNA. Furthermore, the co-expressed mRNAs of IncRNAs were identified by calculating the Pearson's correlation $(\mathrm{P}<0.05)$. The functional enrichment terms for annotating the co-expressed mRNAs were determined using the hypergeometric cumulative distribution function $(17,18)$. The top 200 reliable prediction associations between the IncRNAs and the predicted functional terms were selected to reflect the functional distribution of the dysregulated lncRNAs. The frequency of each predicted functional term for these associations was determined, following which the GO term and KEGG term with more functional annotations were statistically identified (19).

Identification of cis-regulated mRNAs for the dysregulated lncRNAs. The present study further examined how the dysregulated lncRNAs may exert activities through cis- and/or trans-regulated mRNAs. The regions of cis-regulation were identified as follows: Gene locations for different lncRNAs on the chromosome were determined; for each dysregulated lncRNA, the mRNAs were identified as cis-regulated mRNAs when the co-expressed mRNA loci were within $300 \mathrm{kbp}$ downstream or upstream of the given lncRNA and the Pearson's correlation of IncRNA-mRNA expression was significant at the $\mathrm{P}<0.05$ level. When the mRNAs did not conform to the cis-regulated mRNA rules, they were identified as possible trans-regulated mRNAs.

Identification of transcription factors associated with dysregulated lncRNAs. It has been documented that specific lncRNAs may be involved in certain biological processes, including transcriptional regulation, through key transcription factors (TFs) (17). Therefore, the TF/chromatin regulation complexes that may have critical co-regulatory roles with lncRNAs were identified $(18,20)$. Briefly, the set of co-expressed mRNAs for IncRNA and TF/chromatin regulation complex target genes was determined, the enrichment level of which was analyzed using the hypergeometric distribution. Therefore, the TFs prominently associated with dysregulated lncRNAs were finally determined. The lncRNA-TF network was established using the hypergeometric distribution, and a graph showing the associations between TFs and IncRNAs was drawn using Cytoscape software (version 3.6.1; http://www.cytoscape.org/), an open source software platform. In the network, the core $\mathrm{TF}$ with the highest degree of expression was considered the centre of highest importance.

\section{Results}

General expression profiles of dysregulated lncRNAs and $m R N A s$. To investigate the lncRNA landscape involved in ATRA-induced APL differentiation, lncRNA and mRNA microarray analyses were performed on BMMCs from patients with APL. The microarray data were filtered through a volcano plot to determine the dysregulated lncRNAs and mRNAs in BM samples from patients with APL post-induction and corresponding BM samples from patients with APL at diagnosis (Fig. 1A). The lncRNA and mRNA expression data were clustered using Cluster 3.0 (Fig. 1B). Based on the similar expression patterns, the samples were further classified into two groups using dendrogram-based methods for clustering. It was found that $825 \operatorname{lncRNAs}$ were dysregulated between the patients with APL post-induction and the matched controls at diagnosis, with 410 upregulated and 415 downregulated lncRNAs (Fig. 1C). Among the dysregulated lncRNAs, NONHSAT076891 was upregulated the most, with an FC of 304.00, whereas TCONS_00022632-XLOC_010933 was downregulated the most, with an FC of 447.09. It was also found that 1,218 mRNAs were dysregulated, with 660 upregulated and 558 downregulated mRNAs (Fig. 1C). The most upregulated and downregulated mRNAs were MPEG1 and CYTL1, with FCs of 257.13 and $1,169.37$, respectively. The top 20 upregulated and downregulated lncRNAs and mRNAs are listed in Table III.

Verification of dysregulated expression of lncRNAs and $m R N A s$. To verify the dysregulated expression of lncRNAs and mRNAs associated with ATRA-induced APL differentiation, RT-qPCR analysis was performed to examine the upregulation or downregulation of the genes. A total of 10 dysregulated lncRNA transcripts were selected for RT-qPCR analysis. These 10 lncRNAs consisted of four randomly selected lncRNAs, including NONHSAT121385, ENST00000469418, ENST00000424415, and NR_003186, and six specifically selected lncRNAs, including ENST00000536425, NONHSAT061249, TCONS_00017553XLOC_008249, TCONS_12_00030950-XLOC_12_015963, NONHSAT076891, and TCONS_00022632-XLOC_010933. ENST00000536425 and NONHSAT061249 were selected as a result of their predicted cis-regulating potential. TCONS_00017553-XLOC_008249 and TCONS_ 12_00030950-XLOC_12_015963 were selected due to their predicted trans-regulating potential and their presence among the top 100 lncRNA-TF pairs. NONHSAT076891 and TCONS_00022632-XLOC_010933 were selected as they were the most upregulated and downregulated, respectively (Table III). The RT-qPCR results were in agreement with the results obtained from the microarray chip analysis (Fig. 2). As shown in Fig. 2, all selected lncRNAs were dysregulated and exhibited the same trend of upregulation or downregulation $(\mathrm{P}<0.05$ for each $\operatorname{lncRNA}$, Student's t-test).

IncRNA and mRNA co-expression profiles and lncRNA function prediction. Several hundred lncRNAs were co-expressed with hundreds of mRNAs. For example, ENST00000419668 was co-expressed with 515 mRNAs and TCONS_00022632-XLOC_010933 with 488 mRNAs. The GO and KEGG pathway annotations of the co-expressed mRNAs were used to predict the functions of the dysregulated lncRNAs. The lncRNAs were clustered into hundreds of GO and KEGG terms with more functional annotations. In the corresponding association between the 'IncRNA name' 
A

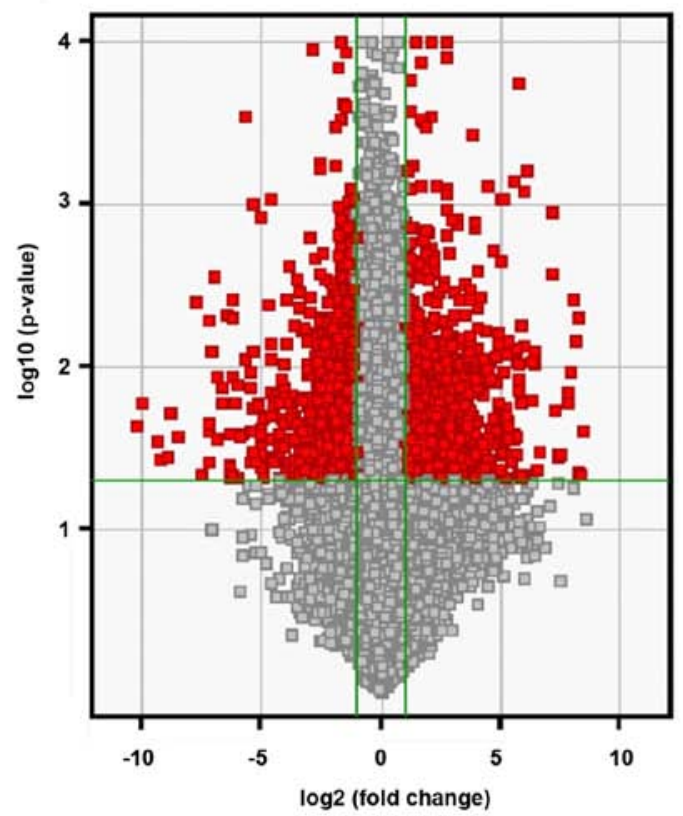

C

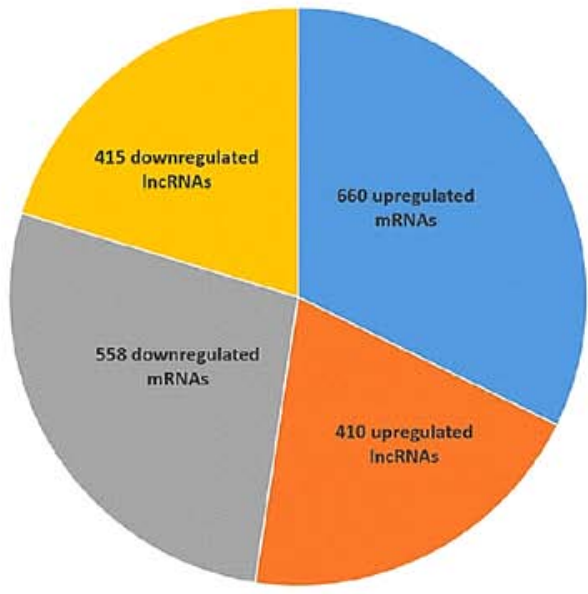

$\mathrm{B}$

\begin{tabular}{|c|c|c|c|c|c|}
\hline-2.0 & & & & & 2.0 \\
\hline 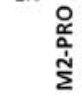 & 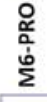 & 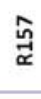 & $\begin{array}{l}\stackrel{u}{\frac{\alpha}{\alpha}} \\
\dot{\vec{d}} \\
\Sigma\end{array}$ & 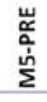 & 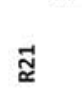 \\
\hline
\end{tabular}

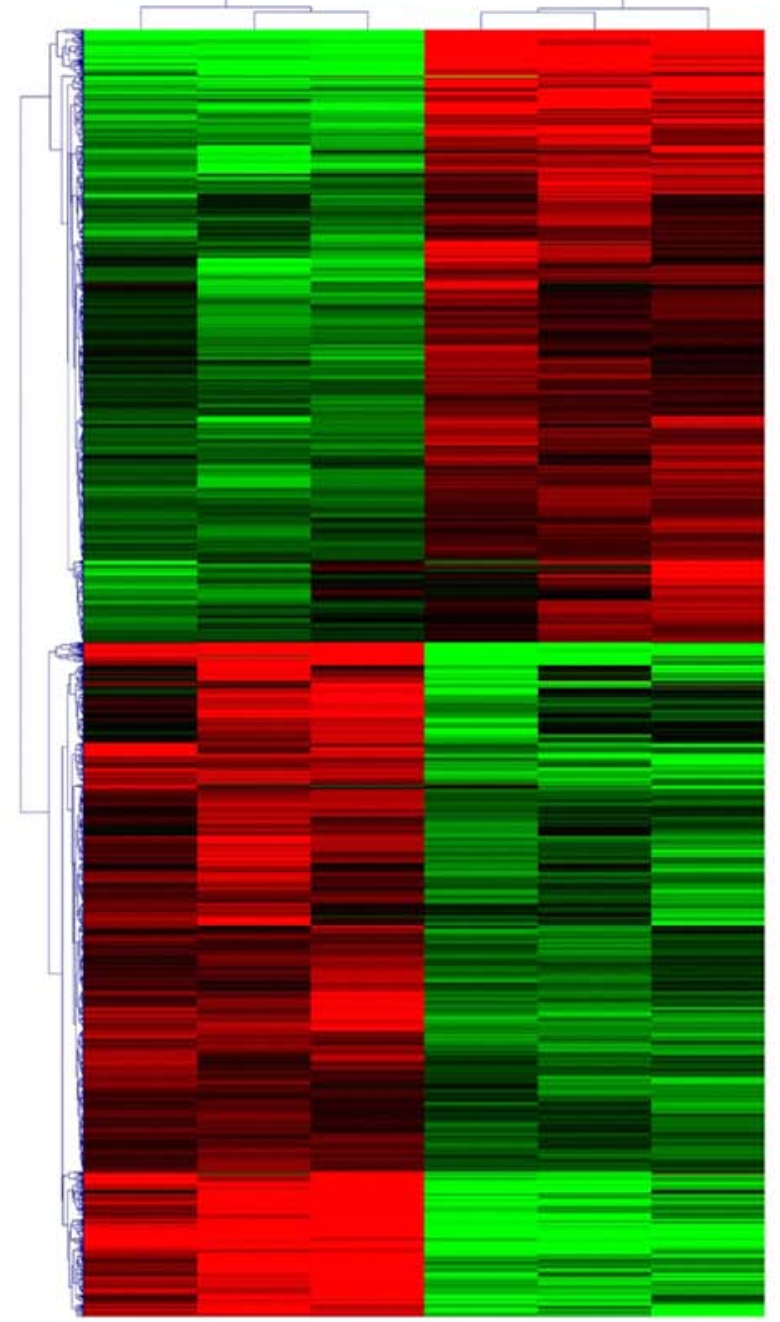

Figure 1. Profiles of dysregulated lncRNAs and mRNAs in BM samples from patients with APL post-induction and corresponding BM samples from patients with APL at diagnosis (pre-induction). (A) Volcano plot of the dysregulated lncRNAs and mRNAs. (B) Heat map of dysregulated lncRNAs and mRNAs. (C) Numbers of common upregulated or downregulated lncRNAs and mRNAs. lncRNAs, long non-coding RNAs; mRNAs, messenger RNAs; BM, bone marrow; APL, acute promyelocytic leukemia.

and 'functional prediction term', the top 200 predicted associations were selected to reflect the functional distribution of the dysregulated IncRNAs. Among the GO terms, the most common biological processes for the dysregulated lncRNAs were cell cycle phase transition, regulation of spindle checkpoint, negative regulation of viral genome replication, DNA damage checkpoint, and attachment of spindle microtubules to kinetochore (Fig. 3A). The most common KEGG terms were DNA replication, spliceosome, $\mathrm{NF}-\kappa \mathrm{B}$ signalling pathway, mismatch repair, primary immunodeficiency, nucleotide excision repair, and cell cycle (Fig. 3B). Therefore, according to the enrichment, cell cycle phase transition was the most enriched GO term and DNA replication was the most enriched KEGG term (Fig. 3).
Analysis of 'cis' IncRNAs and their adjacent co-expressed $m R N A s$. Evidence shows that a number of lncRNAs may cis-regulate the transcription of themselves and their adjacent mRNAs by recruiting remodelling factors to local chromatin (21). To determine the potential 'cis' mRNAs associated with a specific lncRNA, the co-expressed mRNAs $300 \mathrm{~kb}$ upstream and downstream of the dysregulated lncRNAs were analyzed. In total, 48 lncRNAs and their predictively cis-regulated mRNAs were identified using accurate genomic mapping based on the criteria mentioned above. The lncRNAs and their underlying cis-regulated mRNAs are listed in Table IV. The results suggested that lncRNA ENST00000536425 cis-regulates one mRNA, early endosome antigen 1 (EEA1) (Fig. 4A), whereas 


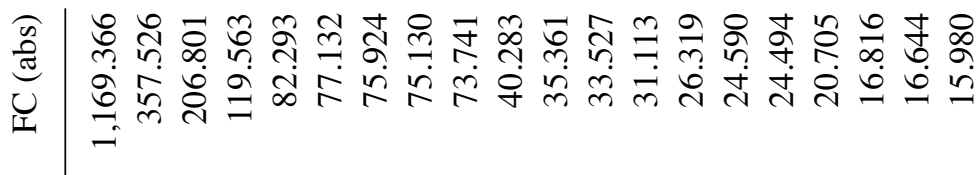

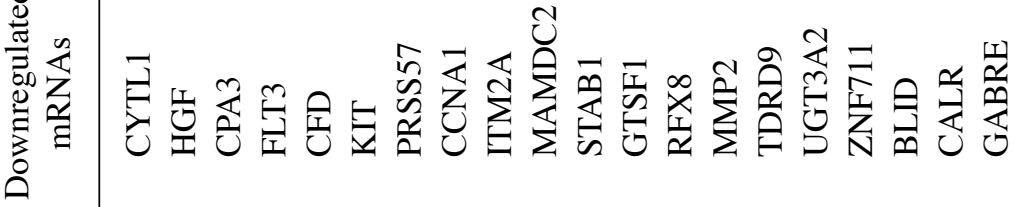

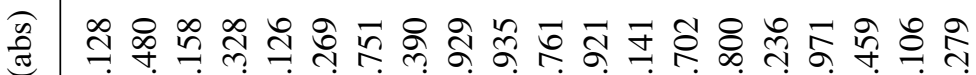

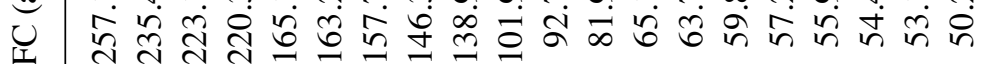

D্ّ 焉艺

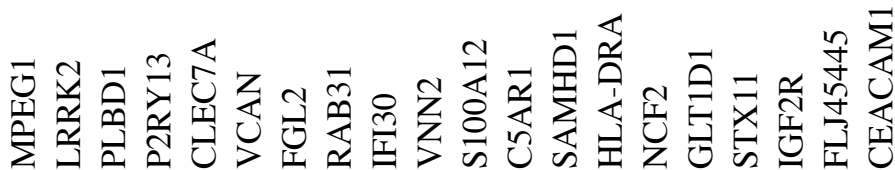
ชิ

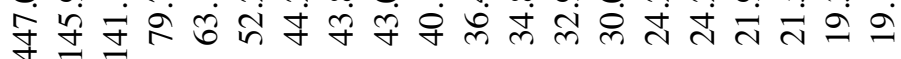

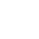

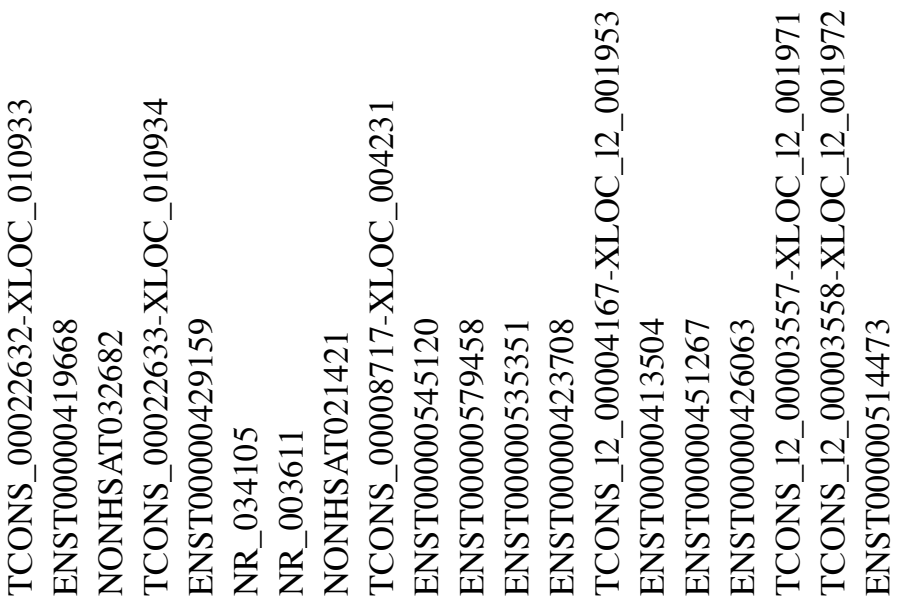

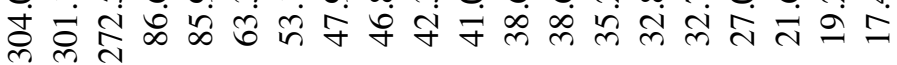




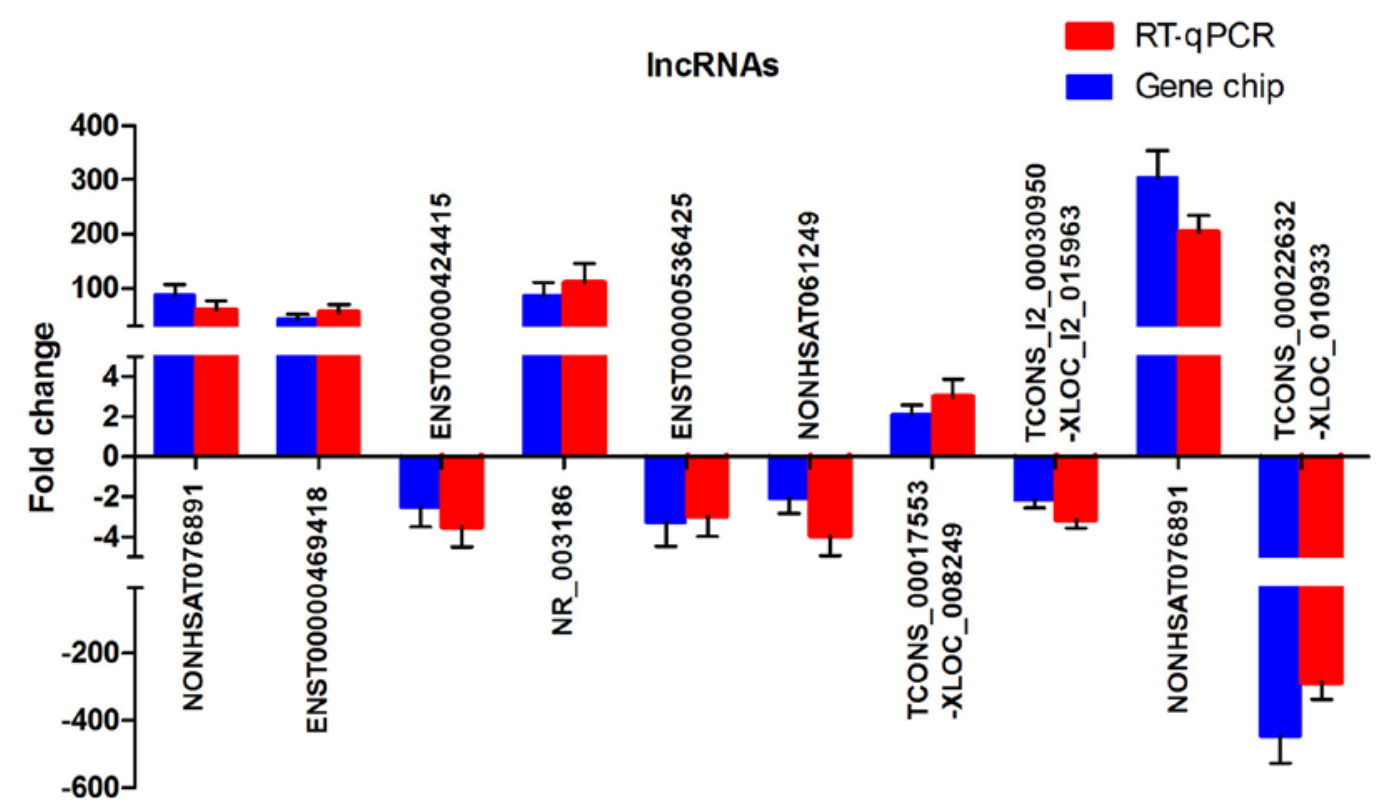

Figure 2. RT-qPCR verification of 10 selected lncRNAs in an additional 27 samples from patients with APL, including 27 post-induction BM samples and 27 pre-induction BM samples. IncRNA expression was consistent with the microarray data ( $\mathrm{P}<0.05$, Student's t-test). IncRNAs, long non-coding RNAs; APL, acute promyelocytic leukemia; RT-qPCR, reverse transcription-quantitative polymerase chain reaction; BM, bone marrow.

A

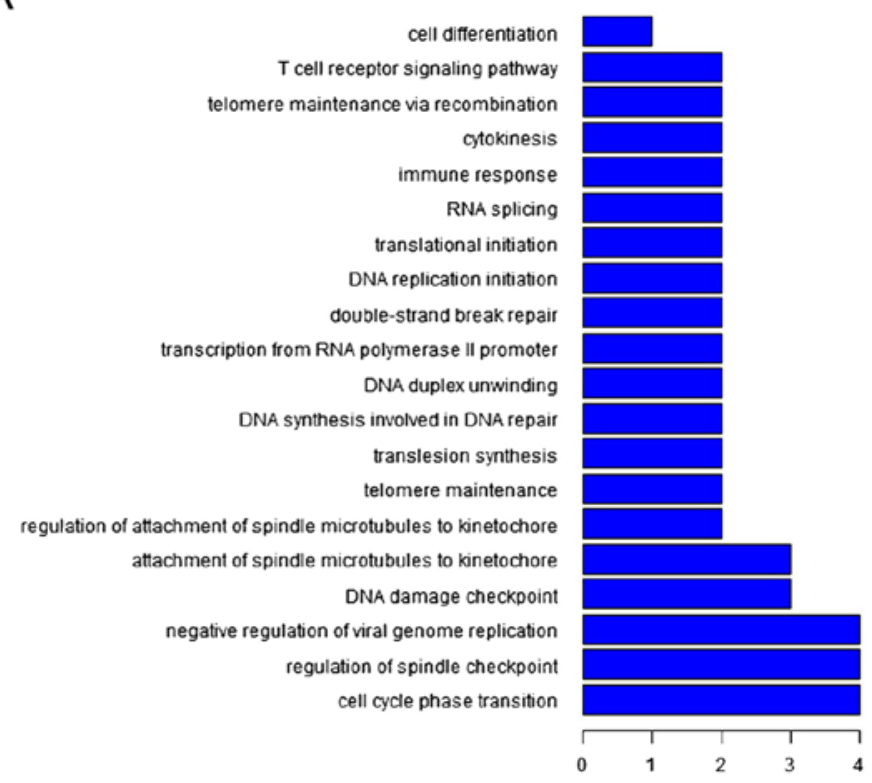

B

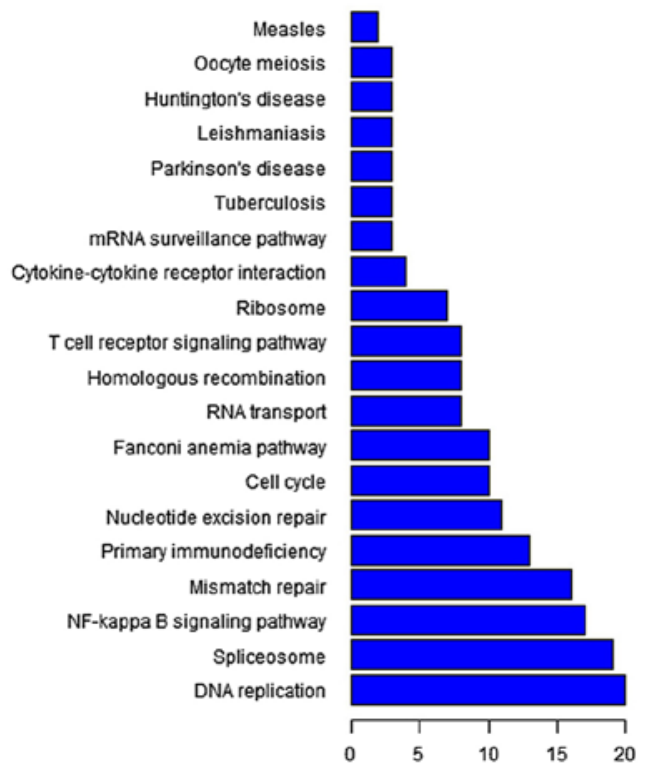

Figure 3. Top 200 GO terms and KEGG pathway terms for dysregulated lncRNAs in BM samples from patients with APL post-induction and BM samples from patients with APL at diagnosis. (A) Top 200 GO term hits for dysregulated lncRNAs. (B) Top 200 KEGG pathway term hits for dysregulated lncRNAs. The $\mathrm{x}$-axis represents the number of annotated lncRNAs, the y-axis represents the GO or KEGG pathway annotations. GO, Gene Ontology; KEGG, Kyoto Encyclopedia of Genes and Genomes; lncRNAs, long non-coding RNAs; BM, bone marrow; APL, acute promyelocytic leukemia.

lncRNA NONHSAT061249 cis-regulates two mRNAs, including zinc finger protein 564 (ZNF564) and zinc finger protein 44 (ZNF44) (Fig. 4B).

Dysregulated lncRNA 'trans' mechanism and construction of the TF-lncRNA-target gene network. As numerous dysregulated lncRNAs were involved in mRNA regulation, a 'TF-lncRNA' network is likely to be large and complex. Consequently, the top 100 associations were selected to generate a core TF-lncRNA network, visualized by hypergeometric distribution analysis (Fig. 5). The core TF-lncRNA network map for patients with APL post-induction, vs. matched controls at diagnosis is shown in Fig. 5A. The majority of potential trans-regulation lncRNAs were found to be involved in pathways regulated by three TFs, including E2F transcription factor 1 (E2F1), E2F transcription factor 6 (E2F6), and early B cell factor 1 (EBF1) (Fig. 5B). In the core network of IncRNA-TF pairs, E2F1, E2F6, and EBF1 regulated the expression of 16 lncRNAs, 13 lncRNAs and 10 lncRNAs, respectively. 
A

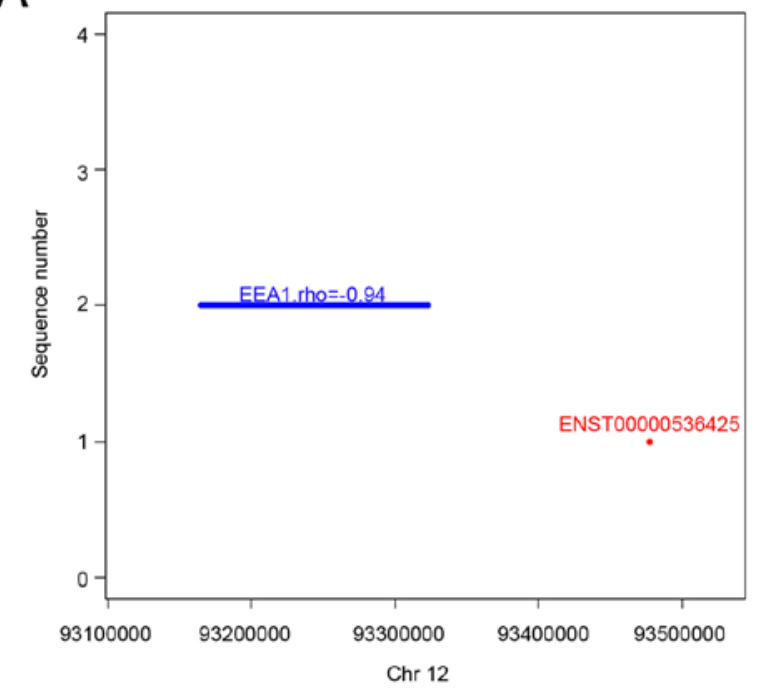

B

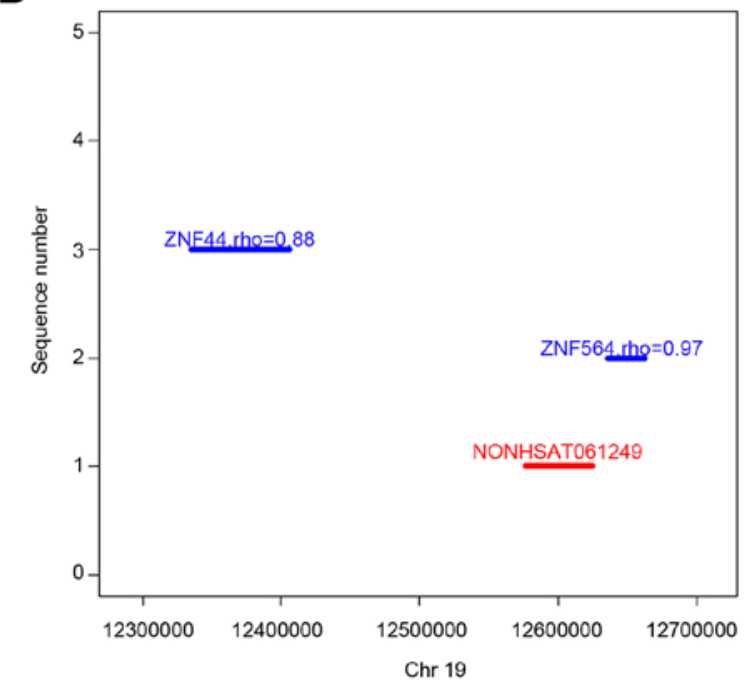

Figure 4. Representative lncRNAs, their 'cis' mRNAs, and their positions on chromosomes. (A) Co-expression of ENST00000536425 and downstream EEA1 mRNA on chromosome 12. (B) Co-expression of NONHSAT061249 and downstream ZNF564 or ZNF44 mRNA on chromosome 19. The abscissa shows the genomic position, the red line/point indicates lncRNA genomic position, the blue line indicates the mRNA location, the rho value represents the correlation coefficients between the lncRNA and its 'cis' mRNA. IncRNAs, long non-coding RNAs; mRNAs, messenger RNAs; EEA1, early endosome antigen 1; ZNF564, zinc finger protein 564; ZNF44, zinc finger protein 44.

A

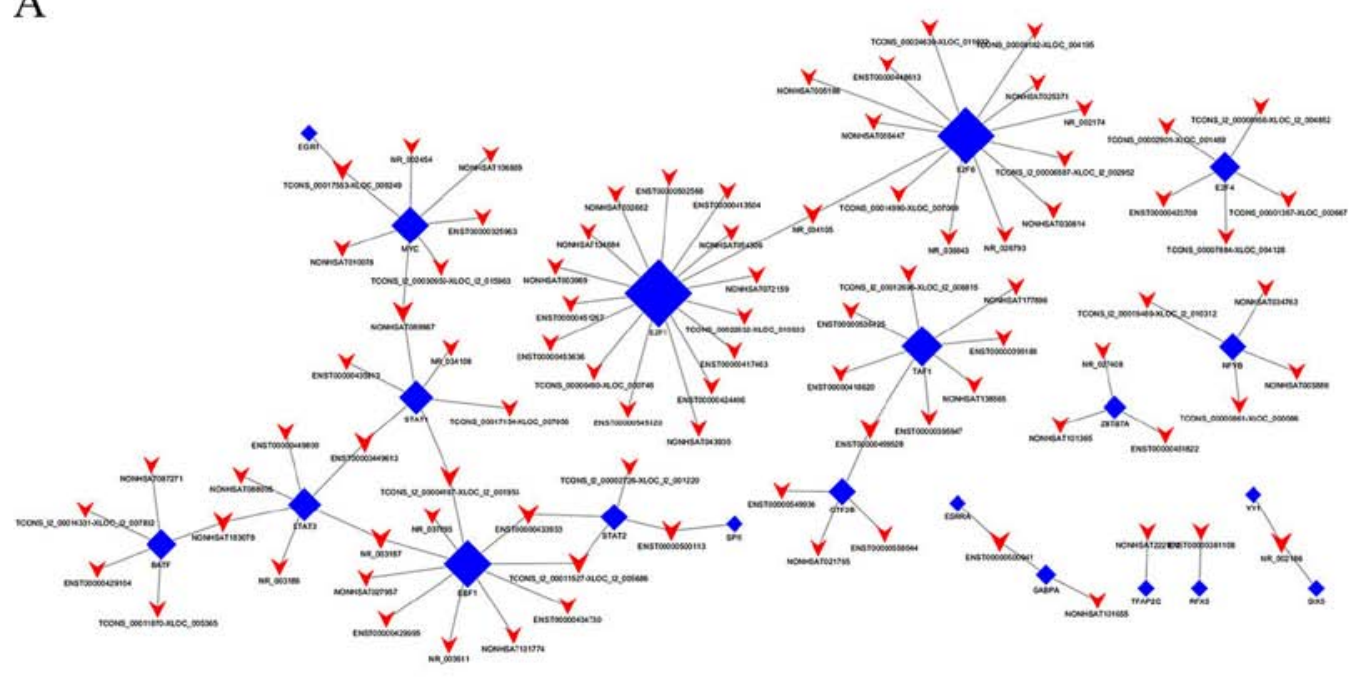

B

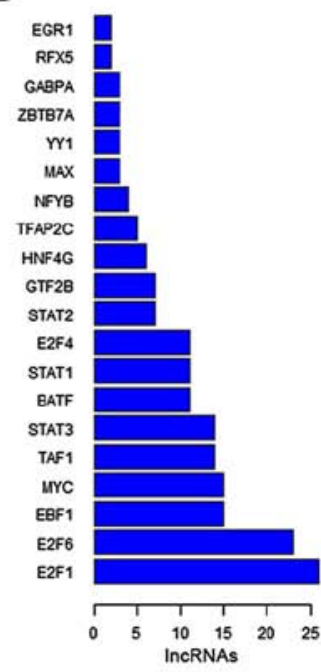

Figure 5. IncRNA-TF core network map for patients with APL post-induction, vs. matched controls at diagnosis. (A) Top 100 lncRNA-TF associations were selected to construct a dyad relationship network using the hypergeometric distribution. Red and blue nodes represent the lncRNAs and TFs, respectively; the node sizes vary as the outlet connection. (B) Majority of lncRNAs were predictively regulated by E2F1, E2F6 and EBF1. The X-axis represents the number of enriched lncRNAs to each TF and the y-axis represents the TF name. IncRNA, long non-coding RNA; TF, transcription factor; APL, acute promyelocytic leukemia; E2F1, E2F transcription factor 1; E2F6, E2F transcription factor 6; EBF1, early B cell factor 1.

Based on the IncRNA co-expression results, the target genes were incorporated into a 'TF-lncRNA' network to determine the TF-lncRNA-target network. Due to the large and complex networks, the top 10 associations were selected to produce a core TF-lncRNA-target network map (Fig. 6). The core TF-lncRNA-target gene association for patients with APL post-induction therapy, vs. pre-induction therapy is shown in Fig. 6, and includes 10 dysregulated lncRNAs (TCONS_12_00006587-XLOC_ $12 \_002952$, ENST00000536425, NR_002174, TCONS_00002901-XLOC_001489, ENST00000558044,
NONHSAT101365, TCONS_00017553-XLOC_008249, TCONS_00000490-XLOC_000746, NONHSAT034763, and TCONS_12_00030950-XLOC_12_015963), 247 target genes, and eight TFs (TAF1, GTF2B, E2F4, ZBTB7A, E2F1, NFYB, MYC, and E2F6) in the core map. As shown in Fig. 6, the core TF $M Y C$ association regulated two lncRNAs (TCONS_0 0017553-XLOC_008249 and TCONS_12_00030950-XLOC_ 12_015963) and 97 target genes. As observed for 'MYCTCONS_00017553-XLOC_008249-RIOK1' in this map, target genes, including $R I O K 1$, were co-expressed for TCONS_00017553-XLOC_008249. As observed for 


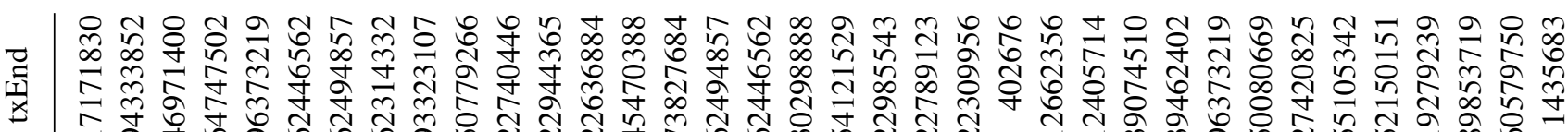

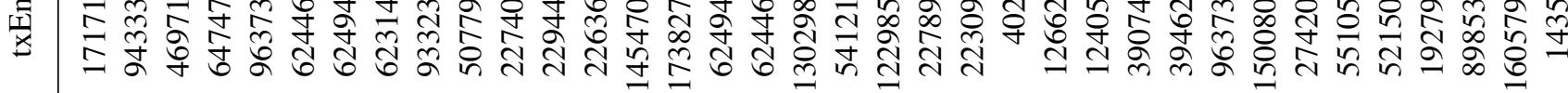

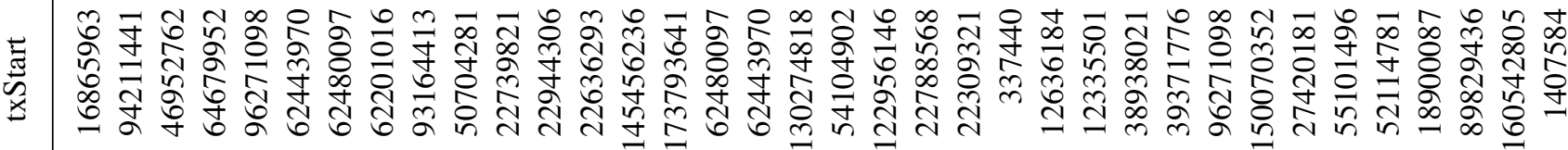

有

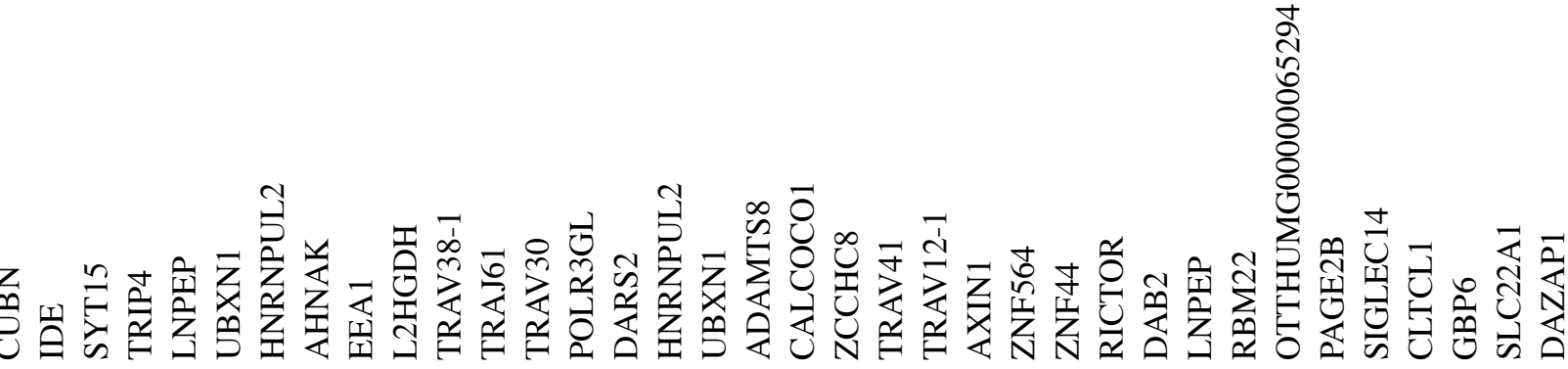

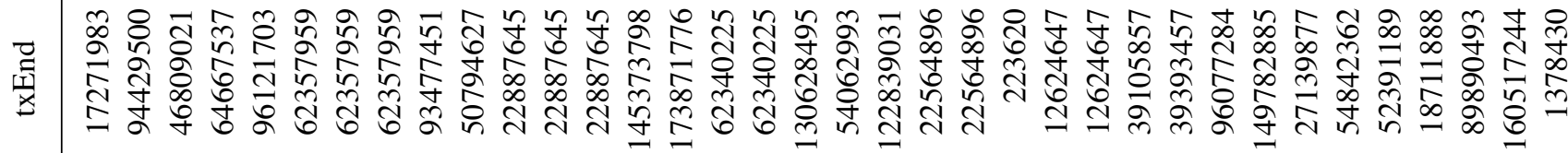

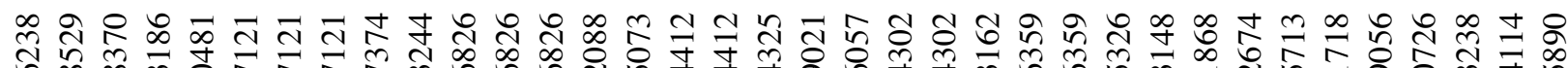

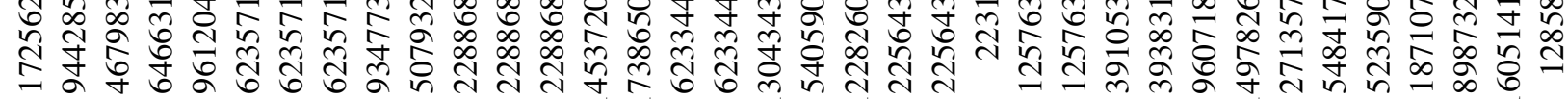

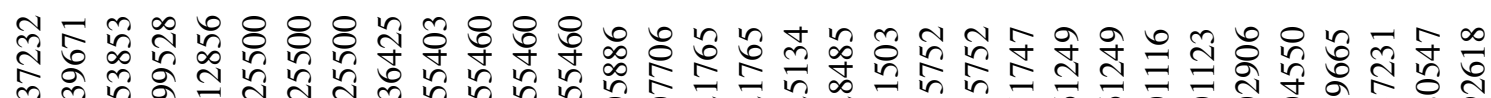

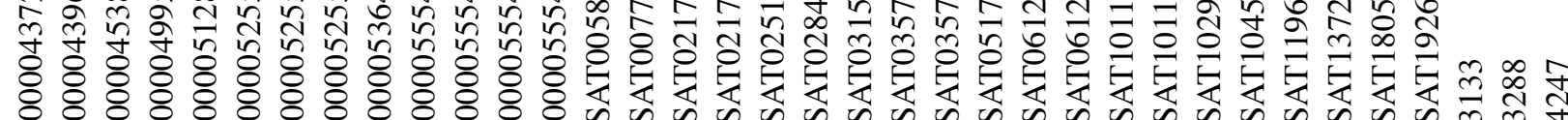

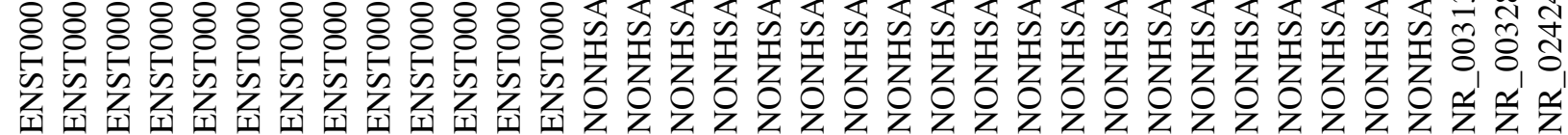

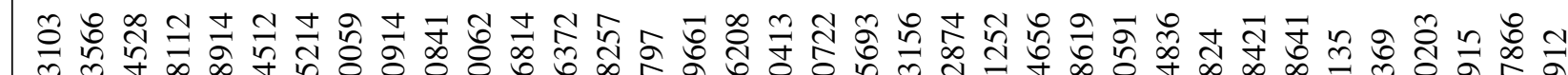

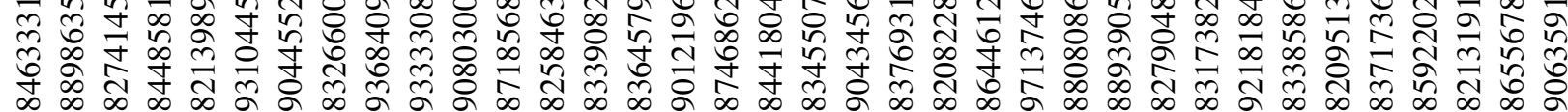

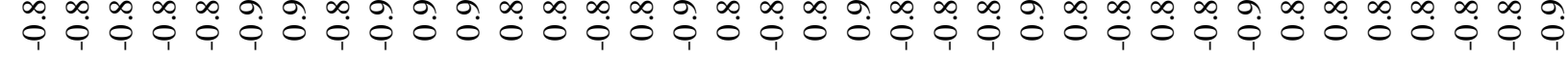
(1) 


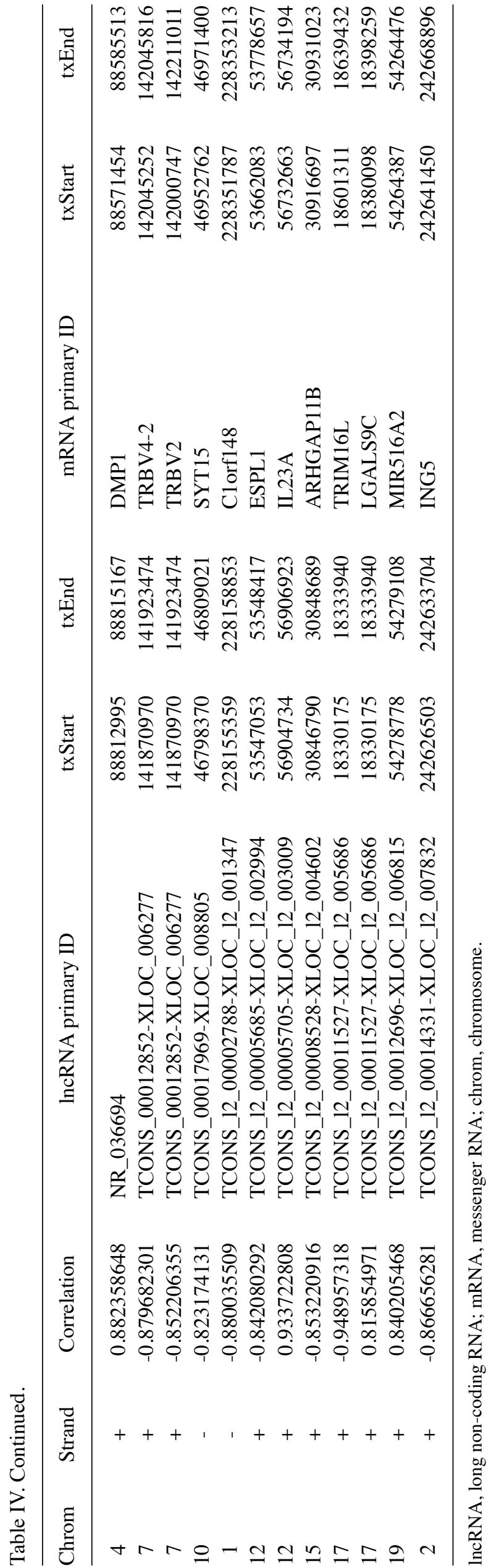

'MYC- TCONS_12_00030950-XLOC_12_015963-RPLP2' in the map, target genes, including $R P L P 2$, were co-expressed for TCONS_12_00030950-XLOC_12_015963-RPLP2. Therefore, these maps provided vital information on the lncRNAs, TFs and target genes.

\section{Discussion}

In the present study, the expression patterns of genome-wide lncRNAs were first evaluated in BM samples from patients with APL post-induction and corresponding BM samples from patients with APL at diagnosis using microarray analysis. Their potential functions were then examined by analysing their co-expressed mRNAs. The experimental results showed that $825 \mathrm{lncRNAs}$ and 1,218 mRNAs were dysregulated. Furthermore, 10 selected dysregulated IncRNAs were validated by RT-qPCR analysis. Several hundred lncRNAs were co-expressed with hundreds of mRNAs, and a number of these may contribute to ATRA/ATO-induced intracorporeal myeloid differentiation by affecting these mRNAs in trans and/or in cis. The present study not only clarified the contributions of lncRNAs to myeloid differentiation in APL and/or intracorporeal therapy response, but also elucidated possible dysregulated lncRNA expression mechanisms associated with ATRA-induced APL differentiation.

APL has shifted from a complex problem in the past into a paradigm with successful targeted therapies, and a series of published randomized clinical trials in patients with APL have all demonstrated efficacy on the frontline of ATRA/ATO association. The identification of a novel class of lncRNAs has attracted attention and may have encouraged investigations focused on characterizing lncRNAs associated with myeloid differentiation and responses to APL therapy. For example, several lncRNAs, including NEAT1 and HOTAIRM1, are indispensable during APL differentiation induced by ATRA $(10,12,22)$. However, numerous 1 ncRNAs and their roles in APL differentiation remain to be fully elucidated. A systematic analysis of the ATRA/ATO-induced intracorporeal myeloid differentiation transcriptome may reflect the complicated and dynamic intracorporeal synergy between ATRA and ATO in patients with APL. IncRNA and mRNA analysis in BM from patients with APL is expected to reflect real changes.

To examine global lncRNA and mRNA profiling in the present study, the Human Transcriptome Array 2.0 was used to screen the dysregulated lncRNAs in three patients with APL post- and pre-induction therapy. The results showed that 825 lncRNAs and 1,218 mRNAs were dysregulated, including 410 upregulated lncRNAs, 415 downregulated lncRNAs, 660 upregulated mRNAs, and 558 downregulated mRNAs, suggesting that these dysregulated IncRNAs may be involved in ATRA-induced myeloid differentiation. Consequently, the present study may assist in determining whether these dysregulated lncRNAs and mRNAs can be applied for the early assessment of APL therapy response or efficacy. The present study also cross-validated the dysregulated gene results from the GeneChip with the results of RT-qPCR analysis; this comparison showed consistency between the microarrays and RT-qPCR results, which supports further predictions. 


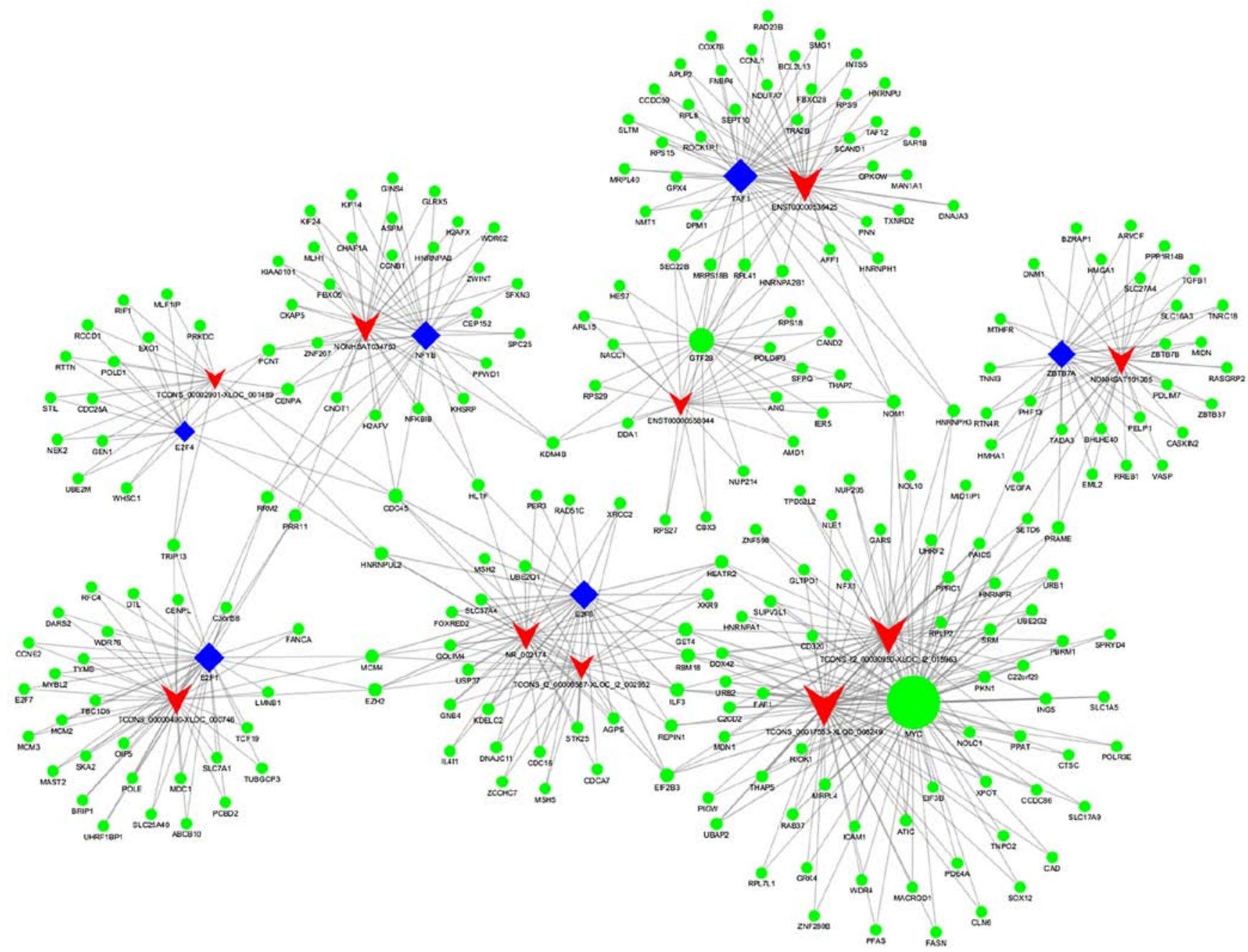

Figure 6. IncRNA-TF-target core network map for patients with APL post-induction, vs. matched controls at diagnosis. The TF-lncRNA-target network comprised 10 lncRNAs, 247 target genes and eight TFs. Blue, red and green nodes represent the TFs, lncRNAs and target genes, respectively; the node sizes vary as the outlet connection. IncRNA, long non-coding RNA; TF, transcription factor; APL, acute promyelocytic leukemia.

An increasing number of lncRNAs have been recognized as critical factors in gene programs to control cell differentiation and function, and serving as scaffolds or decoys at the transcriptional and translational levels (23). Although the number of novel and known lncRNAs is increasing exponentially, only a small subset of these have functional annotations, and the most commonly used method to predict lncRNA function is by investigating their co-expressed mRNAs and associated biological pathways. In the present study, it was found that thousands of mRNAs are co-expressed with the dysregulated lncRNAs. Through functional prediction with the co-expressed mRNAs, the present study identified lncRNAs involved in DNA replication associated with cell cycle phase transition as the most affected by ATRA-induced myeloid differentiation. When stabilized and inhibited via cell cycle phase transitions, lncRNAs function as a vital factor for regulating cell cycle during the course of myeloid maturation in NB4 APL cells (13). Therefore, IncRNAs may be a potential modulator of DNA replication, in addition to certain regulatory TFs.

Compared with mRNAs, lncRNAs have the inherent characteristic of cis-regulation (24) and can cis-regulate their adjacent mRNAs (25). The epigenetic upregulation of
lncRNAs is associated with cis-downregulation of a functional gene cluster in leukemia (26). In the present study, 48 lncRNAs were found to potentially promote cis-regulation of their adjacent mRNAs. Although the majority of the identified lncRNAs have not been characterized, two sets of lncRNA-mRNAs, including ENST00000536425 and EEAI mRNA and NONHSAT061249 and ZNF564 and ZNF44 mRNAs, were identified. A previous study revealed that EEAl serves as an identifying marker of early endosomes for neddylation of type II receptor, and aberrant neddylation results in the development of leukemia (27). The biological processes associated with ZNF564 and ZNF44 are involved in transcription and transcription regulation, respectively. These findings suggest that lncRNA-mRNA networks may contribute to the regulation of cell responses to ATRA-induced APL differentiation.

Certain IncRNAs have been demonstrated to be involved in cis-regulation; however, the majority of characterized lncRNAs are functionally trans-regulating $(18,24)$. The present study predicted the trans-regulatory functions of IncRNAs through TFs. In the central network of IncRNA-TF pairs, the potential trans-regulatory lncRNAs were mainly trans-regulated by E2F1, E2F6, and EBF1. E2F1 and E2F6 are TFs 
that contribute to controlling cell cycle. Several studies have associated the activity of E2F with cell-cycle control $(28,29)$. The E2F1-C/EBPa feedback loop regulates the expression of the oncogene tribbles 2, which is important for AML cell proliferation control (30). E2F6 may transcriptionally regulate cell-cycle G1/S genes via recruiting BRG1 (29). EBFl, a TF that is critical for normal and malignant B-lymphocyte development, controls DNA repair in a dose-dependent manner, which may explain the reason for the frequent loss of the $E B F 1$ gene in leukemia (31). In the present study, the dysregulated lncRNAs involved in the pathways mainly regulated by E2F1, E2F6, and EBF1 were candidate participants in ATRA-induced myeloid differentiation. Consequently, the 'trans' analysis provides a method to interpret the functions of lncRNAs and their biological processes in APL therapy.

The lncRNA-TF analysis identified novel lncRNAs and three TFs for enriched dysregulated mRNAs that contributed to APL treatment. The results of the 'cis' and 'trans' analyses provided essential clues on the modular regulation of lncRNAs. The data obtained may promote future investigations on therapeutic mechanisms in APL. However, the present study had several limitations. First, the sample size was small; thus, investigations with larger sample sizes are required. Second, as the functions of several of the identified lncRNAs have not been annotated, it was only possible to predict the functions of IncRNAs through network and pathway analyses with their co-expressed mRNAs. Therefore, the biological functions of these IncRNAs require further validation.

In conclusion, the present study offered insight into the genome-wide patterns of lncRNA expression during the course of ATRA-based APL therapy. A set of dysregulated IncRNAs were identified in patients with APL who received ATRA-based therapy compared with untreated matched controls. Several lncRNAs may be involved in biological pathways associated with ATRA-induced myeloid differentiation through the cis and/or trans regulation of mRNAs. Furthermore, targeting aberrantly activated pathways in APL cells may offer strategies to circumvent or mitigate disease. The present study provides a foundation for future investigations on lncRNAs associated with ATRA-based APL therapy as therapeutic and diagnostic targets by supplying candidate genes. Additionally, the results provide a platform for systematically evaluating a large number of lncRNAs and mRNAs to identify pathways critical for APL elimination using primary APL patient specimens prior to and following targeted therapy.

\section{Acknowledgements}

We thank Professor Yan Li and Professor Yongqing Tong (Department of Clinical Laboratory, Renmin Hospital of Wuhan University) for their selfless assistance. We thank Shanghai OE Biotech Co., Ltd. (Shanghai, China) for providing lncRNA chip analyses and American Journal Experts (AJE) for providing language help.

\section{Funding}

The present study was supported by the Medical and Health Research Science and Technology Plan Project of Zhejiang
Province (grant nos. 2017KY112 and 2018KY523), the Public Welfare Science and Technology Plan Project of Wenzhou City (grant no. Y20170201), the National Nature Science Foundation of China (grant no. 81701426) and the PhD Research Launching Fund Project of the Second Affiliated Hospital of Wenzhou Medical University (grant no. FEY001).

\section{Availability of data and materials}

The datasets used during the present study are available from the corresponding authors upon reasonable request.

\section{Authors' contributions}

ZGC, JCR and XQZ designed and supervised the project. YYB and JJY provided the samples and clinical information. JY and XLG performed the experiments. YYB and XQZ provided instructions for the experiments. ZGC and JY analyzed the data and wrote the manuscript. ZGC and XLG edited the manuscript. All authors read and approved the manuscript and agree to be accountable for all aspects of the research in ensuring that the accuracy or integrity of any part of the work are appropriately investigated and resolved.

\section{Ethics approval and consent to participate}

Written informed consent was collected from all the patients in conformity with the Declaration of Helsinki, and the present study obtained permission from the Ethics Committee of Wenzhou Medical University.

\section{Patient consent for publication}

Written informed consent was collected from all the patients.

\section{Competing interests}

The authors declare that they have no competing interests.

\section{References}

1. Ablain J, Rice K, Soilihi H, de Reynies A, Minucci S and de Thé H: Activation of a promyelocytic leukemia-tumor protein 53 axis underlies acute promyelocytic leukemia cure. Nat Med 20: 167-174, 2014.

2. Li K, Wang F, Cao WB, Lv XX, Hua F, Cui B, Yu JJ, Zhang XW, Shang S, Liu SS, et al: TRIB3 promotes APL progression through stabilization of the oncoprotein PML-RAR $\alpha$ and inhibition of p53-mediated senescence. Cancer Cell 31: 697-710 e697, 2017.

3. Wang ZY and Chen Z: Acute promyelocytic leukemia: From highly fatal to highly curable. Blood 111: 2505-2515, 2008.

4. Lo-Coco F, Avvisati G, Vignetti M, Thiede C, Orlando SM, Iacobelli S, Ferrara F, Fazi P, Cicconi L, Di Bona E, et al: Retinoic acid and arsenic trioxide for acute promyelocytic leukemia. $\mathrm{N}$ Engl J Med 369: 111-121, 2013.

5. Lehmann-Che J, Bally C and de Thé H: Resistance to therapy in acute promyelocytic leukemia. N Engl J Med 371: 1170-1172, 2014.

6. Zhu HH, Qin YZ and Huang XJ: Resistance to arsenic therapy in acute promyelocytic leukemia. N Engl J Med 370: 1864-1866, 2014.

7. Wang $\mathrm{KC}$ and Chang HY: Molecular mechanisms of long noncoding RNAs. Mol Cell 43: 904-914, 2011.

8. Pan JQ, Zhang YQ, Wang JH, Xu P and Wang W: lncRNA co-expression network model for the prognostic analysis of acute myeloid leukemia. Int J Mol Med 39: 663-671, 2017. 
9. Cao L, Xiao PF, Tao YF, Hu SY, Lu J, Zhao WL, Li ZH, Wang NN, Wang J, Feng X, et al: Microarray profiling of bone marrow long non-coding RNA expression in Chinese pediatric acute myeloid leukemia patients. Oncol Rep 35: 757-770, 2016.

10. Chen ZH, Wang WT, Huang W, Fang K, Sun YM, Liu SR, Luo XQ and Chen YQ: The IncRNA HOTAIRM1 regulates the degradation of PML-RARA oncoprotein and myeloid cell differentiation by enhancing the autophagy pathway. Cell Death Differ 24: 212-224, 2017.

11. Wei S, Zhao M, Wang X, Li Y and Wang K: PU.1 controls the expression of long noncoding RNA HOTAIRM1 during granulocytic differentiation. J Hematol Oncol 9: 44, 2016.

12. Zeng C, Xu Y, Xu L, Yu X, Cheng J, Yang L, Chen S and Li Y: Inhibition of long non-coding RNA NEAT1 impairs myeloid differentiation in acute promyelocytic leukemia cells. BMC Cancer 14: 693, 2014.

13. Zhang X, Weissman SM and Newburger PE: Long intergenic non-coding RNA HOTAIRM1 regulates cell cycle progression during myeloid maturation in NB4 human promyelocytic leukemia cells. RNA Biol 11: 777-787, 2014.

14. Zheng PZ, Wang KK, Zhang QY, Huang QH, Du YZ, Zhang QH, Xiao DK, Shen SH, Imbeaud S, Eveno E, et al: Systems analysis of transcriptome and proteome in retinoic acid/arsenic trioxide-induced cell differentiation/apoptosis of promyelocytic leukemia. Proc Natl Acad Sci USA 102: 7653-7658, 2005.

15. Sanz MA, Grimwade D, Tallman MS, Lowenberg B, Fenaux P, Estey EH, Naoe T, Lengfelder E, Büchner T, Döhner H, et al: Management of acute promyelocytic leukemia: Recommendations from an expert panel on behalf of the European LeukemiaNet. Blood 113: 1875-1891, 2009.

16. Schmittgen TD and Livak KJ: Analyzing real-time PCR data by the comparative C(T) method. Nat Protoc 3: 1101-1108, 2008.

17. Guttman M, Amit I, Garber M, French C, Lin MF, Feldser D, Huarte M, Zuk O, Carey BW, Cassady JP, et al: Chromatin signature reveals over a thousand highly conserved large non-coding RNAs in mammals. Nature 458: 223-227, 2009.

18. Guttman M and Rinn JL: Modular regulatory principles of large non-coding RNAs. Nature 482: 339-346, 2012.

19. Gerstein MB, Kundaje A, Hariharan M, Landt SG, Yan KK, Cheng C, Mu XJ, Khurana E, Rozowsky J, Alexander R, et al: Architecture of the human regulatory network derived from ENCODE data. Nature 489: 91-100, 2012.
20. Khalil AM, Guttman M, Huarte M, Garber M, Raj A, Rivea Morales D, Thomas K, Presser A, Bernstein BE, van Oudenaarden A, et al: Many human large intergenic noncoding RNAs associate with chromatin-modifying complexes and affect gene expression. Proc Natl Acad Sci USA 106: 11667-11672, 2009.

21. Guenzl PM and Barlow DP: Macro lncRNAs: A new layer of cis-regulatory information in the mammalian genome. RNA Biol 9: 731-741, 2012.

22. Wang Y, Fu L, Sun A, Tang D, Xu Y, Li Z, Chen M and Zhang G: C/EBP $\beta$ contributes to transcriptional activation of long non-coding RNA NEAT1 during APL cell differentiation. Biochem Biophys Res Commun 499: 99-104, 2018.

23. Alvarez-Dominguez JR and Lodish HF: Emerging mechanisms of long noncoding RNA function during normal and malignant hematopoiesis. Blood 130: 1965-1975, 2017.

24. Lee JT: Epigenetic regulation by long noncoding RNAs. Science 338: 1435-1439, 2012.

25. Ørom UA, Derrien T, Beringer M, Gumireddy K, Gardini A, Bussotti G, Lai F, Zytnicki M, Notredame C, Huang Q, et al: Long noncoding RNAs with enhancer-like function in human cells. Cell 143: 46-58, 2010.

26. Garding A, Bhattacharya N, Claus R, Ruppel M, Tschuch C, Filarsky K, Idler I, Zucknick M, Caudron-Herger $M$, Oakes C, et al: Epigenetic upregulation of lncRNAs at 13q14.3 in leukemia is linked to the In Cis downregulation of a gene cluster that targets NF-кB. PLoS Genet 9: e1003373, 2013.

27. Zuo W, Huang F, Chiang YJ, Li M, Du J, Ding Y, Zhang T, Lee HW, Jeong LS, Chen Y, et al: c-Cbl-mediated neddylation antagonizes ubiquitination and degradation of the TGF- $\beta$ type II receptor. Mol Cell 49: 499-510, 2013.

28. Attwooll C, Lazzerini Denchi E and Helin K: The E2F family: Specific functions and overlapping interests. EMBO J 23: 4709-4716, 2004.

29. Leung JY and Nevins JR: E2F6 associates with BRG1 in transcriptional regulation. PLoS One 7: e47967, 2012.

30. Rishi L, Hannon M, Salomè M, Hasemann M, Frank AK, Campos J, Timoney J, O'Connor C, Cahill MR, Porse B and Keeshan K: Regulation of Trib2 by an E2F1-C/EBP $\alpha$ feedback loop in AML cell proliferation. Blood 123: 2389-2400, 2014.

31. Prasad MA, Ungerbäck J, Åhsberg J, Somasundaram R, Strid T, Larsson M, Månsson R, De Paepe A, Lilljebjörn H, Fioretos T, et al: Ebf1 heterozygosity results in increased DNA damage in pro-B cells and their synergistic transformation by Pax5 haploinsufficiency. Blood 125: 4052-4059, 2015. 